\title{
Addressing COVID-19 Challenges in a Randomized Controlled Trial on Exercise Interventions in a High- Risk Population
}

\section{Gunver Kienle ( $\square$ gunver.kienle@uniklinik-freiburg.de)}

Medical Center - University of Freiburg, Faculty of Medicine https://orcid.org/0000-0001-9498-5944

\section{Paul Werthmann}

Medical Center - University of Freiburg, Faculty of Medicine

\section{Birgit Grotejohann}

Medical Center - University of Freiburg, Faculty of Medicine

Theodor Hundhammer

Eurythmy4you.com

\section{Claudia Schmoor}

Medical Center - University of Freiburg, Faculty of Medicine

Christoph Stumpe

Shen Men Institut

\section{Sebastian Voigt-Radloff}

Medical Center - University of Freiburg, Faculty of Medicine

\section{Roman Huber}

Medical Center - University of Freiburg, Faculty of Medicine

\section{Research Article}

Keywords: Telemedicine, e-health, exercise, trials, aged, falls, Tai Chi, eurythmy therapy, chronic disease, COVID-19

Posted Date: October 30th, 2020

DOl: https://doi.org/10.21203/rs.3.rs-100074/v1

License: (c) (1) This work is licensed under a Creative Commons Attribution 4.0 International License. Read Full License 


\section{Abstract}

Background: The coronavirus disease 2019 (COVID-19) pandemic is a threat to ongoing clinical trials necessitating regular face-to-face, in-person meetings, particularly in participants with a high risk of complications. Guidance on how to handle and safely continue such trials is lacking. Chronically ill elderly individuals require-in addition to protection from infection-regular physical exercise and social contacts to remain healthy. Solutions for these conflicting necessities are needed.

Methods: The ENTAIER trial is investigating the influence of mindful movements on fall risk, fear of falling, mobility, balance, quality of life, and other outcomes. The study population consists of 550 chronically ill elderly individuals with a high risk of falling. The movements are regularly performed in coached groups over 6 months. After the trial began, COVID-19 lock-downs stopped all in-person meetings, and it was expected that the limitations of this pandemic would continue long term. Therefore, the exercise program, which involve complex movements and is typically conducted face-to-face in groups, had to be substituted by a telemedicine program within a short timeframe. The objectives, therefore, were to identify challenges and tasks that need to be resolved and steps that need to be taken to achieve high-quality, efficacy, safety, and enable human encounter and motivation.

We proceeded with four steps: 1) A literature review on quality and feasibility issues of telemedicine in general and specifically in exercise training in elderly individuals. 2) Participation in two international telemedicine task forces on integrative medicine, particularly mind-body medicine for cancer patients. 3) A broad discussion and interviews with study therapists,[1] personnel, and international experts on providing mindful movement exercises and other physiotherapies via live telecommunication technology, and with scientists and patient representatives. 4) A review and final evaluation of the core trial team and subsequent planning and implementation of changes.

Results and Conclusions: A variety of tasks and challenges were identified for the technical equipment for therapists and patients, ability of therapists and trial participants to adequately manage the technology and telemedicine intervention, reservations and concerns about the technology among therapists and participants, safety and data protection in using the technology, and study design. Steps and possible solutions led to solutions for both a complete switch to telemedicine and a partial switch in the form of risk management pertaining to the former design and implementation. These steps and possible solutions may also inform other researchers or health care providers confronted with similar challenges in the current situation or similar future scenarios.

[1] For practical purposes, eurythmy therapists and Tai Chi teachers are summarized here as therapists.

\section{Background}

Coronavirus disease 2019 (COVID-19), which is caused by severe acute respiratory syndrome coronavirus 2 (SARS-CoV-2), was first identified in China at the end of 2019 and then rapidly spread to Europe, to the US, and throughout the world. The World Health Organization declared the SARS-CoV-2 outbreak a Public 
Health Emergency of International Concern on 30 January and a pandemic on 11 March. By that time, Europe had become the epicentre of the pandemic. ${ }^{1}$ Until an effective vaccination becomes available, precautions have to be taken to protect the population and, particularly, people with a higher risk of infection. Direct person-to-person transmission, mainly via respiratory droplets, is the primary route of transmission, but transmission may also occur if a person touches a contaminated surface and then touches his or her eyes, nose, or mouth or through aerosols. ${ }^{2,3}$ Therefore, a combination of physical distancing, hand hygiene measures, and mask use in the community is the most effective prevention strategy.

SARS-CoV-2 infection can lead to several degrees of disease, from asymptomatic or mild symptoms in most patients to severe, critical, or lethal disease in a minority of patients. Severe illness predominantly occurs in adults with advanced age or underlying medical comorbidities. ${ }^{2}$ This patient group is advised to take precautions, practice social distancing, and stay at home. ${ }^{4}$ However, limiting physical and social activities and becoming house-ridden impair physical fitness and can accelerate frailty, dependency, and the feeling of isolation and, thus, increase the risk of physical and mental diseases or impairment of chronic diseases; consistent physical exercise and social activity are essential to maintain physical and emotional health and autonomy and to reduce the risk of falling. 5,6

Clinical trials, particularly those investigating physical exercise on-site and in-person in elderly individuals, are facing several challenges during the COVID-19 pandemic and the concomitant need for social distancing, lock-down, and quarantine. Guidance on the management of clinical trials during this public health crisis has been released by the European Medicines Agency (EMA) ${ }^{7}$ and other international bodies. ${ }^{8}$ However, many obstacles and questions regarding the application of high-quality treatment and protocol-compliant clinical trials while protecting participants remain unresolved.

The intensity and duration of constraints during the COVID-19 pandemic and the return to normalcy are only partially predictable. Nevertheless, patient care and clinical trials must continue; thus, possibilities to manage the situation must be developed. A possible solution is telemedicine, which is now being rapidly and globally implemented. However, the development of telemedicine in exercise interventions, particularly in the elderly, is still in its infancy. We found no guiding principles on how to switch large ongoing clinical trials investigating physical exercise in a high-risk population from in-person to telemedicine interventions and how to adapt these trials to the restrictions introduced by the pandemic.

We are currently conducting a large, publicly funded, multicentre clinical trial (the ENTAIER-trial ${ }^{9}$ ), which investigates the efficacy and safety of slow, complex, and mindful movement exercises in chronically ill elderly individuals with a high risk of falling. The trial had just begun and had 12 exercise groups when we had to cancel all in-person meetings and the enrolment of new participants. Herein, we report how we proceeded and found guiding principles for the substitution of the exercise program with a telemedicine program in the pandemic and how to adapt the program to the different abilities, weaknesses, needs, and reservations of the participants. This procedure, the identified steps, and the possible solutions may support other research teams in similar situations.

Page 3/19 


\section{Materials And Methods}

\section{The situation of the ENTAIER trial}

At eight trial sites, 550 outpatients aged 65 years and older with chronic diseases and an increased risk of falling are randomly assigned to participate in 36 group sessions of either eurythmy therapy (EYT) or Tai Chi over 24 weeks or to receive standard care alone. A motivation and communication concept supports the trial participants' compliance, and written and video instructions support home training. The primary outcome is fall risk over 6 months. The secondary outcomes are fear of falling, balance, mobility, cognition, mood, quality of life, instrumental activities of daily living, use of medical and non-medical services, and adherence, which are assessed at 3,6, and 12 months. The trial is funded by the Federal Ministry of Education and Research (BMBF 01GL1805); the trial was registered in the German Clinical Trials Register DRKS00016609 at 30th July 2019. After initiation at three of the eight study sites and recruitment of 99 of 550 participants, the COVID-19 pandemic stopped patient recruitment, study site initiations, group sessions, and visits. The intensity and duration of the COVID-19 pandemic was unpredictable but expected to necessitate precautions and restrictions in the following one to two years. These restrictions were expected to vary across the different study sites. They particularly affect the vulnerable population of chronically ill elderly individuals, some of whom are deeply scared by the situation. To find a solution for this situation, possible modifications of the trial design and treatment application had to be identified that could be applied at all study sites, do not increase the risk of infection among participants, and instill confidence in participants and their relatives so that they could continue to participate even in the event of an unfavourable development regarding COVID-19.

The obvious solution was a switch to telemedicine (i.e., live, synchronous, two-way video-conferencing); however, telemedicine is new in most exercise interventions for elderly individuals with a high risk of falling and is particularly new in the mindful, complex exercises used in our trial, which are usually performed in-person and where direct, face-to-face human interaction is regarded as essential. No guidance exists on how to substitute such a complex movement intervention in elderly individuals for a telemedicine intervention during an ongoing clinical trial. We therefore had to answer the following questions: What challenges and tasks need be resolved? What steps need to be taken? How can highquality, efficacy and safety be achieved? How can we still enable human encounters and motivation when using telemedicine?

\section{Methods}

We conducted a broad inquiry and proceeded with the following four steps: 1) A literature review on quality, ethical, and feasibility issues, guiding principles, clinical trials, and trial protocols for telemedicine in general and specifically in exercise training in elderly individuals. ${ }^{10-35}$ 2) Participation in two international telemedicine task forces on integrative, particularly, mind-body medicine for cancer patients, that included broad discussions of the relevant issues, a broad survey of approximately 50 integrative 
oncology experts from 19 countries, and the development of international guidelines for telemedicine cancer care. 3) In-depth discussions with participating therapists and study personnel of the ENTAIER trial and 11 international experts providing mindful movement exercises, particularly Tai Chi and EYT, and other physiotherapies for elderly individuals via live-streaming internet conference systems with scientists and patient representatives. The procedure was stepwise, meaning that results and topics introduced in the literature review and first discussions were included in subsequent discussions with the experts. 4) The results were listed in a table and evaluated and discussed in the core trial team, including patient representatives and a hygiene expert, in view of new developments in the COVID-19 pandemic.

\section{Results}

Two solutions were identified: a complete switch to telemedicine elements and risk management implementation in the primary design.

The main challenges and tasks identified were related to issues with a) technology for therapists and participants; b) the ability of therapists and participants to handle the technique and media-mediated interventions; $c$ ) reservations and concerns about the technique among therapists and participants; and d) safety and data protection in dealing with technology.

\section{Plan 1: Complete switch to telemedicine}

The literature and discussions with experts showed that live, synchronous, online interventions were feasible, appeared to be effective and safe, were well received by participants and therapists, and seemed to enable human encounters. Still, experiences in chronically ill elderly individuals with a high risk of falling are limited. The advantage of telemedicine is independence from locally varying developments during the pandemic and respective varying regulations.

To ensure high quality, safety, feasibility, and adaptation to the abilities of the elderly, a variety of challenges and tasks as well as possible solutions were identified and are summarized in Table 1. They have implications for study design, study sites, and study organization in addition to general trial adaptions (e.g. visits) as recommended by EMA ${ }^{7}$ (Table 2).

\section{Table 1: Tasks, Challenges, Solutions and Steps for a Complete Switch to Telemedicine in the ENTAIER- Trial}




\begin{tabular}{|c|c|c|}
\hline Issue & Task, Challenge & Solution, Steps \\
\hline \multirow{8}{*}{$\begin{array}{l}\text { Technology } \\
\text { for } \\
\text { therapists }\end{array}$} & \multirow{8}{*}{$\begin{array}{l}\text { Older people have limitations in seeing, } \\
\text { hearing, and processing information, and } \\
\text { special requirements are necessary for the } \\
\text { technical equipment used by therapists. }\end{array}$} & $\begin{array}{l}\text { - High resolution streaming } \\
\text { camera. }\end{array}$ \\
\hline & & $\begin{array}{l}\text { - Good illumination in the } \\
\text { therapist's room, with 6-8 light } \\
\text { sources (softboxes) and no } \\
\text { shadows. }\end{array}$ \\
\hline & & - Bluetooth headset. \\
\hline & & $\begin{array}{l}\text { - Sufficient space so the therapist } \\
\text { can step back far enough and still } \\
\text { be seen from head to toe without } \\
\text { distractions in the room; room wide } \\
\text { and deep enough so sidewalls are } \\
\text { not visible. }\end{array}$ \\
\hline & & $\begin{array}{l}\text { - Clothing that allows the } \\
\text { movements to be seen in high- } \\
\text { contrast and does not match the } \\
\text { room colour; no distraction due to } \\
\text { other items (e.g. plants). }\end{array}$ \\
\hline & & $\begin{array}{l}\text { - Additional screens that allow the } \\
\text { therapist to clearly see the patients. }\end{array}$ \\
\hline & & $\begin{array}{l}\text { - Support by a second person at } \\
\text { least in the first } 3-5 \text { hours. }\end{array}$ \\
\hline & & $\begin{array}{l}\text { - Due to investment in equipment } \\
\text { and training, possibly only } 3-4 \\
\text { therapists per arm, central facilities } \\
\text { used by several individuals, or } \\
\text { rental of appropriately equipped } \\
\text { rooms. }\end{array}$ \\
\hline \multirow[t]{5}{*}{$\begin{array}{l}\text { Technology } \\
\text { for patients }\end{array}$} & \multirow[t]{3}{*}{$\begin{array}{l}\text { Older people are only partly equipped with } \\
\text { smart electronic devices with video } \\
\text { capabilities suitable for video-conferencing } \\
\text { (e.g. computer, laptop, or tablet with } \\
\text { microphone, speakers, and webcam). }\end{array}$} & $\begin{array}{l}\text { - Recommend support and supply } \\
\text { of technical equipment from } \\
\text { relatives (e.g. children or } \\
\text { grandchildren), neighbours, or } \\
\text { friends. }\end{array}$ \\
\hline & & $\begin{array}{l}\text { - Rental tablets for participants } \\
\text { who do not have a smart electronic } \\
\text { device. }\end{array}$ \\
\hline & & - Pre-setting the tablet. \\
\hline & \multirow[t]{2}{*}{$\begin{array}{l}\text { Elderly individuals may not have access to } \\
\text { reliable internet or Wi-Fi. }\end{array}$} & $\begin{array}{l}\text { Rental tablet equipped with a } \\
\text { SIM card that allows internet } \\
\text { access. }\end{array}$ \\
\hline & & - Adaption of inclusion criteria. \\
\hline Handling the & Little telemedical experience. & - Training therapists for one day \\
\hline
\end{tabular}


with the target population and with the therapist as a test patient to have his or her own experience.

- Therapists should know each other and regularly communicate.

The focus of teaching shifting from primarily visual to primarily verbal instructions.

Digital perception of the patient and his or her strengths, limitations, risks, personality is impaired compared to in-person meetings.

- Therapists need to be able to teach with a verbal rather than a purely visual teaching style.

- Prior digital consultation of each patient or consultation during the first 1-3 group sessions.

- Additional screens (see above).

Handling the technique participants
Little experience and competence with internet, video-conferences, mobile devices, or desktop computer.

- Train children, grandchildren, or neighbours to assist and support participants with the use of internet, video-conferences, and computers.

- Easy-to-use and intuitive online meeting tool.

- Good instruction given to participants at the study centre verbally, written, and step-by-step.

- Telephone support.

The interventions are complex and new for participants; the exercises and instructions in the video-conference may not be as easy to understand as in an in-person meeting; individual adaptation may not be as precise; the written manual and audio-visual instructions initially supplied to the participants may be too demanding, especially in the beginning.

Reservations about the technique by therapists
Emotional reservations, concerns regarding human encounters, and spiritual aspects.
- Start slow. Easy instructions. Ask questions for understanding. Record every group session (the therapist), and provide the video for one week or until the next lesson (in addition to the written manual and the audio-visual instructions given to the participants).
- Specifically addressing the topic and related sub-aspects, exchanging among therapists and the trial team, lectures by experts trusted as authorities, and training.

- Research question for qualitative sub-study and focus group.

Negative attitude towards the medium may induce nocebo effects.
- Therapists must have a positive attitude towards the medium.

- Instructions for positive communication through a communication coach. Put participants at ease when they feel insecure about technology or some technical problems arise. Create a pleasant atmosphere. 
Disappointment of therapists who cannot participate.
- Discussions with therapists who are already included, explaining the current situation of the COVID-19 pandemic.

\section{Reservations about the} technique by participants
Emotional reservations and human aspects.
- Assisted test video call with familiar persons (e.g. grandchild or child) during the baseline visit to the study centre.

- Explanation of the possibility to stay connected with close ones during lock-down, quarantine, etc.

- Communication suggestion for therapists to approach the patient, create a pleasant atmosphere, convey empathy, paraphrase questions, summarise the response, ask about participants' feelings and experiences with video-conferences, and ask for feedback.
Safety of Participants are at risk of falling and possibly participants fragile; multitasking is difficult for them.
- Safe space (size, no trip hazards, and stable chair) and checklist for safety aspects.

- Instructing children, grandchildren, neighbours, or friends to assist, train, and supervise in the first three hours.

- Clear view of the participants (technical equipment) and clear instructions by therapists (verbal teaching style).

- Therapists need to estimate the ability of participants and the group to multitask and, if necessary, rearrange the training so that participants initially only watch the exercise and subsequently perform the exercise without looking at the screen. Train therapists for this situation.

Sufficient space
- Adapt the inclusion criteria for sufficient space.

- Individual adaption of exercises.

- Start each class by checking the room for trip hazards.

- Check the necessity for additional videos and instructions and of recording therapist instructions. 
Data safety During the video-conferences, other participants and the therapists can see patients and the private room of the patients (and record it if this is intended).

Data protection or safety issues of the provider.
- Inform the participants to arrange their clothing and the section in the apartment seen in the conference, accordingly.

- Promote privacy and confidentiality.

- Investigate the data protection or safety issues of the provider. Consult a data protection officer.

Table 2: Additional Tasks for the Study Team 


\begin{tabular}{|c|c|c|}
\hline Issue & Necessity, Challenge & Solution, Task \\
\hline $\begin{array}{l}\text { Study } \\
\text { design }\end{array}$ & $\begin{array}{l}\text { Additional questions regarding } \\
\text { - Feasibility (technology). } \\
\text { - Practicability (users). } \\
\text { - Satisfaction and acceptance } \\
\text { (users). } \\
\text { - Barriers (users and } \\
\text { technology). } \\
\text { - Interaction of patients and } \\
\text { therapists. } \\
\text { - Suitability of and improvement } \\
\text { in the medium for therapists. } \\
\text { - Technology and aspects of } \\
\text { humanity and confidence. } \\
\text { - The degree to which the } \\
\text { therapist and participants } \\
\text { connect during the exercises or } \\
\text { are restrained by the technique. }\end{array}$ & $\begin{array}{l}\text { - Adaptions in trial protocol and patient } \\
\text { information. } \\
\text { - } \quad \text { Additional questions (quantitative analysis). } \\
\text { - } \text { Possible change in the primary objective. } \\
\text { - } \text { Qualitative substudy. } \\
\text { - } \quad \text { Focus group. } \\
\text { - Potential filming of a subgroup for analysis. } \\
\text { - Informed consent of the subgroup. } \\
\text { - } \quad \text { Adaptions in the statistical analysis. }\end{array}$ \\
\hline $\begin{array}{l}\text { Study } \\
\text { centres }\end{array}$ & $\begin{array}{l}\text { - Increased participant need for } \\
\text { information. } \\
\text { - Little experience with media. }\end{array}$ & $\begin{array}{l}\text { - Detailed information for participants, } \\
\text { necessitating more time; repeat of information } \\
\text { when distributing the study book. } \\
\text { - Discussion of hurdles, fears, and barriers; if } \\
\text { necessary, acquaintance with the media (e.g. } \\
\text { test video call with a family member or friend } \\
\text { during the baseline visit). } \\
\text { - Technical instructions (must be repeated); for } \\
\text { some participants, telephone support before the } \\
\text { first group session. } \\
\text { - Loan tablet, if necessary. } \\
\text { - Instruction of assistance at home. }\end{array}$ \\
\hline $\begin{array}{l}\text { Study } \\
\text { organisation }\end{array}$ & $\begin{array}{l}\text { - Study organisation, coordination } \\
\text { items, communication with all parti } \\
\text { questionnaires, protocols, guidance } \\
\text { adaptation according to EMA recon }\end{array}$ & $\begin{array}{l}\text { and management: Planning, organisation or all } \\
\text { es, adaptation of all trial documents, } \\
\text { ethical approval, discussion with sponsor, } \\
\text { mendations. }{ }^{7}\end{array}$ \\
\hline
\end{tabular}




\section{Estimation and recommendation by public and patient representatives}

One of the public and patient representatives supported the necessity of a complete switch to telemedicine. Two others strongly recommended against it, although they saw the necessity to identify alternatives in view of COVID-19; they reported increasing signs of introversion, concern, and anger among elderly individuals. They criticized that the authorities and media would spread fear among elderly individuals and inform them that only contact avoidance would protect them. In addition, elderly individuals had not been consulted for their opinions and needs regarding the measures implemented for their protection. Increasing isolation would imply "social death". The sole option, becoming house-ridden and having television or other digital activities as the main activites, increases the risk of social isolation and physical and emotional inactivity, and this tendency could be enhanced by another telemedicine tool. The patient representatives regarded direct human encounters as essential for life and internal strength of a person. Therefore, they recommended in-person meetings and information, including hygiene concepts, which should be evidence-based and not fear-driven, for the participants. This strategy should enable the elderly to participate in society and life again.

\section{Plan 2: Telemedicine as risk management - the decision for the ENTAIER trial}

We discussed plan 1 within the study organizing team and reassessed the situation of COVID-19 in Germany; a total switch to telemedicine enables group exercise, provides motivation for exercise, and maintains human encounters to minimize feelings of isolation without the risk of infection and without the necessity to wear masks. However, there was agreement that regarding efficacy, safety, and human encounters, physical meetings for exercise are clearly preferable. Exercises are better to perceive, understand, and follow when there is in-person instruction and interaction compared to instructions seen only in a small digital screen and exercises can be better adapted to an individual's strengths and weaknesses in-person. Physical meetings improve human connection and, therefore, motivation to practise exercise. Furthermore, the effort to attend physical meetings with public or private transport may be an additional activating factor, and absolute infection prevention is only achievable with long-term isolation, which may not be in the patient's best interest, as pointed out by patient representatives. Thus, the goal is to avoid increasing the normal risk when participating in social activities following the recommended precautions.

At the time of the evaluation, the COVID-19 situation in Germany had greatly improved and stabilized and seemed to be well handled by the German population and its political and medical authorities. Consequently, the lock-down was reduced, and physical meetings were allowed when hygiene precautions and physical distancing were followed. Thus, physical meetings were less risky, and people increasingly demanded to return to their normal lives, and elderly individuals wanted to participate in social activities. The acceptance of and expected compliance with only telemedical intervention were expected to decrease. Therefore, we reconsidered the situation and decided to proceed with a second 
plan, conducting the trial as before but with the implementation of protective measures and risk management:

Protective measures to reduce the transmission of infection follow the regulations and advice of national and local governments, health authorities, institutions, and relevant guidelines (e.g. social distancing, masks, hygiene, and home isolation after contact with individuals infected with SARS-CoV-2 or when symptoms are present). The risk management strategy evolved from the above listed solutions but was simplified as necessary, considering that the majority of session and, particularly the introductory group sessions, will be held in-person. If, due to the pandemic, meetings are not possible, group classes will switch to telemedicine. Participants, particularly those who are unfamiliar with computers, tablets, or internet-based video-conferencing, will be encouraged to seek support from family members or friends (e.g. test video calls to friends or family) or will be supported by study personnel in advance to familiarize them with the technology and to reduce stress if and when the use of technology is necessary. Participants who participate in Tai Chi or EYT group sessions and can not have their own computer or tablet can borrow a tablet from the trial site free of charge.

An user-friendly internet communication system (e.g. whereby.com or Jitsi Meet), which does not require registration or the entry of any personal data of the participants, will be provided for the groups. Participants will be provided step-by-step written guidance. In case social distancing on a national or regional level will be necessary, group classes can be switched to telemedicine courses (live, synchronous, internet-based video-conferencing or, if not possible despite all efforts, with instructions via telephone) led by the corresponding Tai Chi teacher or EYT therapist. Participants will then be familiar with the exercises, training, and therapist and, therefore, can switch to telemedicine classes more easily. In addition, the therapists will have met the participants, have assessed their strengths and weaknesses, and can guide and tailor the exercises to the participants. In case social distancing on an individual level will be necessary (e.g. quarantine or individual concern), patients across the trial sites will be offered participation in group sessions of their assigned intervention using telemedicine. These sessions will be taught by a therapist with additional expertise in telemedicine and with the appropriate technical infrastructure. It will be documented whether the session was conducted face-to-face, as a videoconference, with verbal telephone instruction only, or not at all. The informed consent form is supplemented with an explanation of the telemedicine risk adjustment. Therapists are instructed on general and technical details of telemedicine, including positive verbal communication to avoid a nocebo effect; they are offered support with information and training. The study sites are informed about the procedure and how to inform and support participants, as well.

If study site visits will temporarily not be possible due to COVID-19, the assessments will be performed via telephone and mail. The Berg Balance Scale and the Montreal Cognitive Assessment will then either be postponed or performed using telemedicine if the patient is accompanied by a person to assist them and to assure their safety. 
This risk adjustment was appreciated by patient representatives and approved by the local ethical committee in Freiburg and have been obtained for the other sites. All methods and procedures were and are carried out in accordance with relevant guidelines and regulations. ${ }^{9}$

\section{Statistical considerations}

As a result of the necessary risk management implementation due to the pandemic, it may happen that patients will not attend face-to-face group classes as originally planned but will attend internet-based video-conferences, receive instructions via telephone, or will receive no teaching at all. The primary analysis of the trial is planned to be performed in all randomised patients regardless of the intensity or type of Tai Chi and EYT teaching which addresses the so-called treatment policy estimand according to the recent ICH E9(R1) guideline. ${ }^{36}$ Consequently, the effect of the mixture of these interventions will be estimated. The size of the expected effect of this mixture compared to the effect of the originally planned face-to-face group classes can only be speculated, but presumably, it will be somewhat smaller. To assess the impact of the restrictions enforced due to the COVID-19 pandemic on the estimated treatment effects, various additional sensitivity analyses are planned. The intensity of the training in each randomised group will be described by the number and type of executed training sessions compared to the planned number of training sessions. Sensitivity analyses will be performed, restricting the population to patients who attended at least $80 \%$ of the originally planned face-to-face training sessions. Additional analyses will be used to describe the effects of changing the type of teaching on the risk of falls.

The aim of the ENTAIER trial is to estimate the effects of Tai Chi and EYT training on the risk of falling over six months without the influence of the COVID-19 pandemic. Therefore, a treatment effect that is not confounded by pandemic-related disruptions should ideally be addressed. In the context of the ICH E9(R1) guideline, ${ }^{36}$ the pandemic will be regarded as an intercurrent event, and a scenario in which the intercurrent event would not occur will be assumed (i.e. addressing the hypothetical estimand). A sensitivity analysis estimating a hypothetical estimand will be performed by disregarding data after faceto-face group classes due to pandemic-related restrictions for an individual patient are stopped. Further sensitivity analyses are conceivable and will be described at a later point in time in the statistical analysis plan, as the duration of the pandemic and its implication on the course of the study are not currently known. EMA's points to consider on the implications of COVID-19 in the methodological aspects of ongoing clinical trials will be considered. ${ }^{7}$

\section{Discussion}

The COVID-19 pandemic introduced restrictions to medical care and clinical trials. Continuity in care and clinical trials are essential and must be guaranteed to prevent any unnecessary health detriment. Thus, the development of e-health, telemedicine, and tele-rehabilitation has accelerated at an extraordinary speed. For instance, in a large New York health system (NYU Langone Health), ambulatory care video encounters increased from $<50$ pre-COVID-19 to more than 7000 within a short timeframe. ${ }^{35}$. Particularly, the physical therapy profession has been thrust into telemedicine and e-health. ${ }^{27}$ Guidance and practical 
tips are published, as well as technical and feasibility issues, ethical and data safety issues, practical issues, clinical trials, and trial protocols. ${ }^{10-28,30-35}$ Collectively, telemedicine encompasses various conditions, populations, and applications and seems to be similar to in-person care. 14,17-19,21,22,30 Participants and caregivers feel comfortable and are satisfied in the short term, even if they have no experience in virtual participation. ${ }^{35} 22,34$ However, many questions regarding high-quality, effective, safe, and motivating mindful exercises provided with telemedicine and ensuring human encounters, particularly in chronically ill elderly individuals, remain open. Therefore, a switch within an ongoing trial, especially in elderly individuals, is particularly challenging.

With the help of the literature and discussions with international experts, we created a list of challenges, tasks, possible solutions, and steps for telemedicine substitution of an exercise intervention for chronically ill elderly individuals with a high risk of falling, which involves mindful, complex movements. Our list may support other researchers and practitioners who are in similar situations, as many of these challenges and tasks apply to many situations of exercise interventions or mind-body medicine that use telemedicine. In addition, guidelines for mind-body cancer care are currently being published, which extend our list, due to the wider issue.

The continuity of physical exercise and social activities during lock-down or quarantine, particularly in elderly individuals, is of utmost importance. A physically inactive lifestyle, social isolation, reduced autonomy, and particularly falls increase the risk for morbidity and mortality. ${ }^{37-41}$ To interrupt this vicious cycle and to enable healthy aging despite chronic disease, it is essential to enhance safe physical, psychological, and cognitive capacities. ${ }^{5,37,38,42}$. Many have, therefore, implemented telemedicine exercise or rehabilitation programs, developed guidance, or are currently testing telemedicine in clinical trials. $5,6,11,16,17,19,21,22,33-35$

A strength of our list is the implementation of wide sources from the literature to the perspectives of interprofessional and international experts experienced in clinical research in the application of different forms of exercise programs, telemedicine, care of chronically ill elderly individuals with physical and partly mental restrictions, and mind-body medicine. This provides a practical list on how to proceed with telemedicine exercise interventions and points out critical issues and challenges. Another strength is the cooperation with patient representatives, who are sometimes not heard and often have a different perspective than clinicians or scientists.

There are also limitations to our list. It is focused on mindful exercises, chronically ill elderly individuals, and the health care situation and population in Germany. Other issues that arise in different types of interventions or other populations in other countries may not have been covered. In addition, the items that are included in our clinical trial protocol in detail, ${ }^{9}$ such as confidentiality, data safety, language barriers, and aspects referring to the details of the intervention, are not listed here. Therefore, they still have to be included in other contexts. Furthermore, we had limited time for our investigation to continue our trial. With more time, we would have extended the expert round and the countries and specialties and could have included a subsequent Delphi technique. However, experience in telemedicine, even by experts, 
remains limited and needs to be improved. Therefore, we might not have gained many more aspects with this approach. Furthermore, we had already included a wide range due to the inclusion of aspects from the literature and due to the participation in two telemedicine task forces, one of which is currently developing guidelines based on a broad survey, and in the last interviews hardly no new aspects were added. Therefore, we presume that we captured most of the important aspects.

Telemedicine will become increasingly important not only due to the pandemic but also to reach patients in remote locations or patients who cannot visit health care providers in person for other reasons. Telemedicine provides a variety of challenges. Therefore, guiding principles, assessments, clinical investigations, and key observations must be published. We recommend including public and patient representatives, as they have unique and important observations of the group of people who will ultimately benefit from telemedicine. Although this inclusion initially seems time-consuming and costly, it may ultimately accelerate and focus processes and prevent detours.

\section{Declarations}

\section{Ethics approval and consent to participate}

Ethics approval from the Committee of the Medical Center - University of Freiburg (183/19; 23.07.2019 and 13.07.2020) and the ethics committees of Tübingen for the Tübingen and Filderstadt sites (561/2019B01, 21.10.2019 and 12.08.2020) of Witten/Herdecke (150/2019, 31.07.2019 and 30.07.2020), Ulm (284/19, 01.08.2019 and 22.07.2020), and Essen (19-8908-BO, 18.02.2020 and 23.07.2020) were obtained. Berlin relies on the vote from Freiburg.

Written informed consent to participate in the study is obtained from all participants.

\section{Consent for publication:}

The interviewed experts gave consent for publishing a summary of the discussed issues and recommendations.

\section{Availability of data and materials}

The principle of open data is supported (https://www.bihealth.org/de/quest-center/missionansaetze/open-science). Anonymised data may be shared with cooperating scientists or scientists who have other medically or scientifically well-founded reasons (goals referring to transparency, replicability, different analyses, combinations and meta-analyses of data). Their research must aim at improving care of elderly people, and they have to ensure data protection.

\section{Competing interests}


The authors declare that they have no competing interests.

\section{Registration and funding}

The trial is registered in the German Clinical Trials Register at www.drks.de, (DRKS00016609) on 30 July 2019.

The trial is funded by the Federal Ministry of Education and Research (BMBF 01GL1805).

\section{Author contributions}

GSK is coordinating investigator and wrote the first draft. PW is medical trial coordinator. BG is the project manager. CS is the trial statistician. SVR serves as a geriatric advisor. $\mathrm{RH}$ is the head of the Center for Complementary Medicine and serves as a medical advisor. TH and ChS serve as specialists in EYT and Tai Chi using telemedicine tools in the ENTAIER trial. All authors substantially contributed to the trial protocol, risk adjustment, and publication. All authors have read and approved the manuscript.

\section{Acknowledgements}

We are grateful to trial doctors, therapists, teachers, patient representatives and to the other trial personnel as well as to the interviewed experts for supporting us, for providing their expertise and participating in the inquiry.

\section{References}

1. WHO. Rolling updates on coronavirus disease (COVID-19). Published 2020. Accessed 01-09-2020.

2. Mclntosh K, Hirsch M, Bloom A. Coronavirus disease 2019 (COVID-19): Clinical features. (C2020 UpToDate, Inc. Wolters Kluwer. Published 2020. Accessed 29-06-2020.

3. Cohen P, Blau J, Elmore J, Kunins G. Coronavirus disease 2019 (COVID-19): Outpatient management in adults. (C2020 UpToDate, Inc. Wolters Kluwer. Published 2020. Accessed 29-06-2020.

4. Centers for Disease Control and Prevention: Checklist for Older Adults. Published 08-05-2020. Accessed 01-09-2020.

5. Aung MN, Yuasa M, Koyanagi Y, et al. Sustainable health promotion for the seniors during COVID-19 outbreak: a lesson from Tokyo. J Infect Dev Ctries. 2020;14(4):328-331.

6. Chevance A, Gourion D, Hoertel N, et al. Ensuring mental health care during the SARS-CoV-2 epidemic in France: A narrative review. Encephale. 2020;46(3):193-201.

7. Guidance on the management of clinical trials during the COVID-19 (coronavirus) pandemic. Published 28 April 2020. Accessed 01-09-2020. 
8. de Paula BHR, Araujo I, Bandeira L, Barreto N, Doherty GJ. Recommendations from national regulatory agencies for ongoing cancer trials during the COVID-19 pandemic. Lancet Oncol. 2020;21(5):624-627.

9. Kienle GS, Werthmann PG, Grotejohann B, et al. A multi-centre, parallel-group, randomised controlled trial to assess the efficacy and safety of eurythmy therapy and tai chi in comparison with standard care in chronically ill elderly patients with increased risk of falling (ENTAiER): a trial protocol. BMC Geriatr. 2020;20(1):108.

10. Dijkstra HP, Ergen E, Holtzhausen L, et al. Remote assessment in sport and exercise medicine (SEM): a narrative review and teleSEM solutions for and beyond the COVID-19 pandemic. Br J Sports Med. 2020.

11. Middleton A, Simpson KN, Bettger JP, Bowden MG. COVID-19 Pandemic and Beyond: Considerations and Costs of Telehealth Exercise Programs for Older Adults With Functional Impairments Living at Home-Lessons Learned from a Pilot Case Study. Phys Ther. 2020.

12. Dorsey ER, Okun MS, Bloem BR. Care, Convenience, Comfort, Confidentiality, and Contagion: The 5 C's that Will Shape the Future of Telemedicine. J Parkinsons Dis. 2020;10(3):893-897.

13. Dorsey ER, Topol EJ. Telemedicine 2020 and the next decade. Lancet. 2020;395(10227):859.

14. Nelson M, Bourke M, Crossley K, Russell T. Telerehabilitation is non-inferior to usual care following total hip replacement - a randomized controlled non-inferiority trial. Physiotherapy. 2020;107:19-27.

15. Hosseiniravandi M, Kahlaee AH, Karim H, Ghamkhar L, Safdari R. Home-based telerehabilitation software systems for remote supervising: a systematic review. Int J Technol Assess Health Care. 2020;36(2):113-125.

16. Ortiz-Pina M, Salas-Farina Z, Mora-Traverso M, et al. A home-based tele-rehabilitation protocol for patients with hip fracture called @ctivehip. Res Nurs Health. 2019;42(1):29-38.

17. Bernocchi P, Vitacca M, La Rovere MT, et al. Home-based telerehabilitation in older patients with chronic obstructive pulmonary disease and heart failure: a randomised controlled trial. Age Ageing. 2018;47(1):82-88.

18. van Egmond MA, van der Schaaf M, Vredeveld T, et al. Effectiveness of physiotherapy with telerehabilitation in surgical patients: a systematic review and meta-analysis. Physiotherapy. 2018;104(3):277-298.

19. Batsis JA, DiMilia PR, Seo LM, et al. Effectiveness of Ambulatory Telemedicine Care in Older Adults: A Systematic Review. J Am Geriatr Soc. 2019;67(8):1737-1749.

20. Ramage ER, Fini NA, Lynch EA, Patterson A, Said CM, English C. Supervised exercise delivered via telehealth in real time to manage chronic conditions in adults: a protocol for a scoping review to inform future research in stroke survivors. BMJ Open. 2019;9(3):e027416.

21. Hong J, Kong HJ, Yoon HJ. Web-Based Telepresence Exercise Program for Community-Dwelling Elderly Women With a High Risk of Falling: Randomized Controlled Trial. JMIR Mhealth Uhealth. 2018;6(5):e132. 
22. Lai B, Bond K, Kim Y, Barstow B, Jovanov E, Bickel CS. Exploring the uptake and implementation of tele-monitored home-exercise programmes in adults with Parkinson's disease: A mixed-methods pilot study. J Telemed Telecare. 2020;26(1-2):53-63.

23. Tousignant $M$, Corriveau $H$, Kairy $D$, et al. Tai Chi-based exercise program provided via telerehabilitation compared to home visits in a post-stroke population who have returned home without intensive rehabilitation: study protocol for a randomized, non-inferiority clinical trial. Trials. 2014;15:42.

24. Tuckson RV, Edmunds M, Hodgkins ML. Telehealth. N Engl J Med. 2017;377(16):1585-1592.

25. van Houwelingen CT, Moerman AH, Ettema RG, Kort HS, Ten Cate O. Competencies required for nursing telehealth activities: A Delphi-study. Nurse Educ Today. 2016;39:50-62.

26. Sharma R, Nachum S, Davidson KW, Nochomovitz M. It's not just FaceTime: core competencies for the Medical Virtualist. Int J Emerg Med. 2019;12(1):8.

27. Lee AC. COVID-19 and the Advancement of Digital Physical Therapist Practice and Telehealth. Phys Ther. 2020;100(7):1054-1057.

28. Nittari G, Khuman R, Baldoni S, et al. Telemedicine Practice: Review of the Current Ethical and Legal Challenges. Telemed J E Health. 2020.

29. Giordano A, Bonometti GP, Vanoglio F, et al. Feasibility and cost-effectiveness of a multidisciplinary home-telehealth intervention programme to reduce falls among elderly discharged from hospital: study protocol for a randomized controlled trial. BMC Geriatr. 2016;16(1):209.

30. Shigekawa E, Fix M, Corbett G, Roby DH, Coffman J. The Current State Of Telehealth Evidence: A Rapid Review. Health Aff (Millwood). 2018;37(12):1975-1982.

31. Chaet D, Clearfield R, Sabin JE, Skimming K, Council on E, Judicial Affairs American Medical A. Ethical practice in Telehealth and Telemedicine. J Gen Intern Med. 2017;32(10):1136-1140.

32. Edmunds M, Tuckson R, Lewis J, et al. An Emergent Research and Policy Framework for Telehealth. EGEMS (Wash DC). 2017;5(2):1303.

33. World Confederation of Physical Therapy, International Network of Physical Therapy Regulatory Authorities: Report of the WCPT/INPTRA Digital Physical Therapy Practice Task Force. Published 2019. Accessed 10.August 2020.

34. Calton B, Abedini N, Fratkin M. Telemedicine in the Time of Coronavirus. J Pain Symptom Manage. 2020;60(1):e12-e14.

35. Mann DM, Chen J, Chunara R, Testa PA, Nov O. COVID-19 transforms health care through telemedicine: Evidence from the field. J Am Med Inform Assoc. 2020;27(7):1132-1135.

36. Agency EM. ICH E9 (R1) addendum on estimands and sensitivity analysis in clinical trials to the guideline on statistical principles for clinical trials. EMA/CHMP/ICH/436221/2017. Published 2017. Accessed 01-09-2020.

37. Böhm K, Tesch-Römer C, Ziese T, (Eds). Gesundheit und Krankheit im Alter. Berlin: Robert Koch Institut;2009. 
38. WHO. World report on Ageing And Health. World Health Organization, Geneve2015.

39. Angevaren M, Aufdemkampe G, Verhaar HJ, Aleman A, Vanhees L. Physical activity and enhanced fitness to improve cognitive function in older people without known cognitive impairment. Cochrane Database Syst Rev. 2008(3):CD005381.

40. Fox KR, Ku PW, Hillsdon M, et al. Objectively assessed physical activity and lower limb function and prospective associations with mortality and newly diagnosed disease in UK older adults: an OPAL four-year follow-up study. Age Ageing. 2015;44(2):261-268.

41. Steptoe A, Shankar A, Demakakos P, Wardle J. Social isolation, loneliness, and all-cause mortality in older men and women. Proc Natl Acad Sci U S A. 2013;110(15):5797-5801.

42. Denkinger MD, Lukas A, Herbolsheimer F, Peter R, Nikolaus T. Physical activity and other healthrelated factors predict health care utilisation in older adults: the ActiFE Ulm study. $Z$ Gerontol Geriatr. 2012;45(4):290-297. 